\title{
Don't stand so close to me: The effect of auditory input on interpersonal space
}

Donna M Lloyd, Anna Coates, Jasmin Knopp, Sarah Oram, Samantha Rowbotham

School of Psychological Sciences, Zochonis Building, University of Manchester, Brunswick Street, Manchester M13 9PL, UK; e-mail: Donna.Lloyd@manchester.ac.uk

Received 30 October 2008, in revised form 3 January 2009

\begin{abstract}
We provide preliminary evidence that listening to music through headphones alters the perception of space around the body - specifically, the interpersonal distance maintained between the self and others. In comparison to an external auditory environment, wearing headphones or earplugs increased the amount of space maintained between the wearer and another person during an active approach paradigm. This finding suggests that, when external cues to spatial location (such as sound) are removed, people compensate by increasing the distance between themselves and others. The implications of this research for navigating busy urban environments and for the social interactions of wearers of personal music systems are discussed.
\end{abstract}

We are all familiar with the sight of people walking along the street or on public transport wearing headphones attached to a personal music system. Anecdotally, the wearers of these devices often seem less engaged in their immediate environment; it is difficult to catch their attention or talk to them and they may not notice when you walk very near to them or they to you. This seeming lack of awareness of other people in their immediate surroundings has been linked with increased crime towards young people wearing mp3 players and prompted safety advice from the UK Home Office and local constabularies ("Don't use headphones while walking-stay alert!"-Home Office communications directorate, November 2004. “...wearing headphones can prevent you from hearing oncoming traffic or someone approaching from behind" - safety advice from Leicestershire Constabulary). The message is clear; in addition to showing you have something to steal, listening to music through headphones also makes you less aware of people in your immediate surroundings, particularly behind you where other people can approach without being seen. Is this purely anecdotal or is there any empirical evidence to support this claim? We aimed to provide preliminary support for the assertion that listening to music through headphones changes the perception and maintenance of distance between oneself and others.

We used a modified version of the 'stop-approach distance' paradigm to measure each participant's interpersonal distance with respect to another person (Greenberg et al 1980). The participant, who was naive with respect to the purpose of the study, was instructed to approach the experimenter (situated at the far end of a $1 \mathrm{~m}$ wide by $3 \mathrm{~m}$ long walkway marked out on the floor with parallel lines of clear sellotape at $10 \mathrm{~cm}$ intervals along its length) and to stop when she reached a distance between herself and the experimenter at which she felt comfortable. This distance was then discreetly recorded by two other experimenters with the sellotape lines used as a scale. Four directions of approach were used: participants either walked with the front of their body facing towards the experimenter (ie facing forwards), or they walked with their back facing towards the experimenter (ie facing backwards), or they walked in a 'crab-like' fashion with either the left or the right side of their bodies facing towards the experimenter. In this way it was possible to measure the distance maintained by the participant between herself and the experimenter at the front, back, left, and right sides of her body. A personal music system with headphones playing 
an unfamiliar acoustic music mp3 file was used to create a personal sound condition not shared between the participant and experimenter ('internal sound'). Foam earplugs with a noise reduction rate of $33 \mathrm{~dB}$ were worn by the participant to create a 'no sound' condition, and background environmental noise that could be heard by both the participant and experimenter created the 'external sound' condition.

Twenty-seven female undergraduate students took part in the experiment. Their ages ranged from 19 to 44 years (mean age $=21$ years, $S D=5.32$ years) and ethical approval was given by the Psychological Sciences Research Ethics Committee of the University of Manchester. The mean distances for all trials are shown in table 1 as a function of auditory condition and approach direction.

Table 1. Mean interpersonal distances, their standard errors (in parentheses), and peripersonal space area for the three auditory conditions separated by approach direction.

\begin{tabular}{|c|c|c|c|c|c|}
\hline \multirow{2}{*}{$\begin{array}{l}\text { Auditory } \\
\text { condition }\end{array}$} & \multicolumn{4}{|c|}{ Approach direction/cm } & \multirow{2}{*}{$\begin{array}{l}\text { Peripersonal } \\
\text { space area } / \mathrm{cm}^{2}\end{array}$} \\
\hline & front & back & left & right & \\
\hline External sound & $56(14)$ & $69(25)$ & 64 (19) & $64(22)$ & 8455 (4607) \\
\hline Internal sound & $61(15)$ & $75(25)$ & $71(20)$ & $69(22)$ & $10049(5162)$ \\
\hline No sound & $61(15)$ & $77(26)$ & 74 (19) & $69(20)$ & 10401 (4937) \\
\hline
\end{tabular}

The data were analysed with an analysis of variance (ANOVA) with the following within-participant factors [auditory condition (3; internal versus external versus no sound) $\times$ approach direction (4; front, back, left, and right)]. The results revealed a main effect of auditory condition $\left(F_{1,26}=6.4, p=0.008\right)$, with significantly smaller distances maintained between the participant and the experimenter under the external sound' condition $($ mean $=63 \mathrm{~cm})$ than under the 'no sound' $($ mean $=70 \mathrm{~cm} ; p=0.003$ ) or 'internal sound' (mean $=69 \mathrm{~cm} ; p<0.001$ ) conditions (ie when the participant was wearing earplugs or headphones playing music, respectively). There was also a main effect of approach direction $\left(F_{1,26}=7.8, p=0.001\right)$, with significantly smaller distances maintained between the participant and the experimenter when the participant approached the experimenter facing forwards $($ mean $=59 \mathrm{~cm}$ ) than under any other approach direction (mean approach distance when facing backwards $=73 \mathrm{~cm} ; p=0.002$; mean approach distance when facing left $=69 \mathrm{~cm} ; p<0.001$; mean approach distance when facing right $=67 \mathrm{~cm} ; p=0.006$; all other comparisons were non-significant $p>0.1$ ). However, there was no interaction between auditory condition and approach direction $\left(F_{1,26}=0.872, p=0.484, \mathrm{~ns}\right)$ or any difference in approach distance between the 'internal' and 'no sound' conditions ( $p=0.575)$.

The area maintained around each individual under the three auditory conditions was calculated by using the formula for calculating the area of a rectangle $1 / 2$ (base $\times$ height). This gave a simple approximation of the size of area of interpersonal space maintained around each participant, similar to the concept of the body buffer zone' (ie "the distance that an organism customarily places between itself and other organisms"; Horowitz et al 1964; Sommer 1959; see figure 1). The results confirmed a significant main effect of auditory condition on area $\left(F_{1,26}=5.1, p=0.021\right)$, with a significantly smaller area maintained around the participant when exposed to conditions of external noise (mean area $=8455 \mathrm{~cm}^{2}$ ) than when wearing earplugs (mean area $=10401 \mathrm{~cm}^{2} ; p=0.01$ ) or headphones playing music (mean area $=10049 \mathrm{~cm}^{2}$; $p<0.001$ ). Again, there was no difference in the area maintained around individuals when listening to music through headphones or wearing earplugs $(p=0.660)$.

In summary, the findings of this simple study indicate there is a link between the use of personal music systems and the perception and maintenance of interpersonal space. 


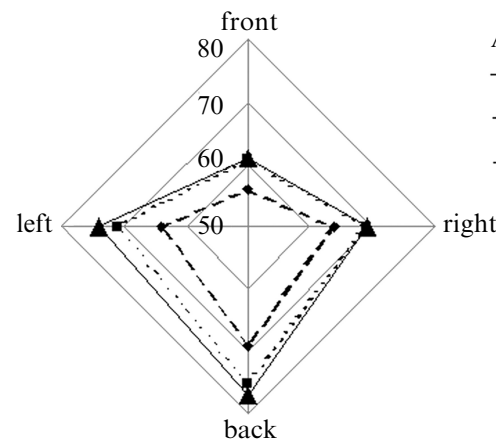

Auditory condition

$-\multimap-$ external sound

-.- internal sound

$\longrightarrow$ no sound

Figure 1. Radar graph illustrating the mean interpersonal distances maintained by participants under each auditory condition. Distances from the experimenter $(\mathrm{cm})$ are shown for the three auditory conditions separated by approach direction (front, back, left, and right).

Listening to music through headphones increased interpersonal distances maintained between the wearer and other people with a trend to suggest this was more apparent in rear space. Because of the decreased availability of external cues, participants appear to have overcompensated by increasing the distance between themselves and the experimenter. Similar research shows that under low-level lighting conditions participants increase the space they leave between themselves and others, and distance requirements increase as the direction of approach progresses from front to rear (Adams and Zuckerman 1991). Recent studies on both healthy participants and neuropsychological patients suggest that audio-somatosensory interactions are greater in rear space, where vision provides no direct information, as people will typically perceive an object's approach from behind by means of auditory cues (see Farnè and Làdavas 2002; Kitagawa et al 2005). Mallenby and Mallenby (1975) demonstrated that hard-of-hearing children who attended a normal public school for a year exhibited similar personal space preferences to those of normalhearing children, whereas those who attended a special institutional school for the deaf showed greater interaction distances during varying dyadic interactions. Taken together these studies suggest that, when external cues to spatial location (such as sound and/or vision) are removed or reduced, participants may compensate by increasing the interpersonal distance between themselves and others, particularly in the space behind the body.

In addition to a reduction in the certainty of auditory cues, the effect of earphones on interpersonal distance may be further mediated by subjective feelings of comfort/ discomfort. A person who is placed in a potentially threatening context will have an expanded personal space (Dosey and Meisels 1969); it is possible that participants in the current experiment felt more disconnected or antisocial during the restricted hearing conditions and were self-consciously keeping their distance from the experimenter. Such defensive-like withdrawing behaviour is thought to be mediated by multimodal cells (receiving visual, tactile, and auditory input) in parietal and prefrontal areas of the brain; the body-centred receptive fields of these cells construct a 'margin of safety' around the individual when an object approaches/enters peripersonal space (Graziano and Cooke 2006; Lloyd 2009). Parietal cells also aid navigation with respect to nearby objects (Bremmer et al 2002) facilitating obstacle avoidance during self-motion. Again, when external sensory cues to interpersonal distance are reduced, these cells may be even more proactive in avoiding impending contact. Future studies would benefit from collecting subjective accounts of participants' awareness of their own personal space and space in relation to the experimenter to address this point.

In modern society mp3 players are commonly worn whilst commuting on public transport or in busy places such as gyms. The wearing of such devices allows the creation of a personal auditory environment that cannot easily be shared by those around (Bull 2000). Although such devices reduce the environmental cues available to the wearer, they may also enable some people to tolerate crowded public environments as the wearer can withdraw from social interaction. We have pilot data suggesting that 
people change their interpersonal space area when listening to music they like compared with music they dislike or no music at all. Listening to self-selected music may make individuals more comfortable and therefore pay less attention to others in the space around them, thus reducing interpersonal distances. Although actual data in support of this assertion are lacking, further empirical research should explicitly address whether people are creating an increased 'sense of space' in crowded urban settings through the use of personal music systems, and whether this is linked with improved mood and decreased social anxiety.

Acknowledgments. We wish to thank Sophia Haythornthwaite for assistance with data collection, and Martin Farrell and an anonymous reviewer for helpful comments on an earlier draft of the manuscript.

\section{References}

Adams L, Zuckerman D, 1991 "The effect of lighting conditions on personal space requirements" Journal of General Psychology $118335-340$

Bremmer F, Duhamel J R, Ben H S, Graf W, 2002 "Heading encoding in the macaque ventral intraparietal area (VIP)" European Journal of Neuroscience $161554-1568$

Bull M, 2000 Sounding Out the City: Personal Stereos and the Management of Everyday Life (Oxford: Berg)

Dosey M A, Meisels M, 1969 "Personal space and self-protection" Journal of Personality and Social Psychology $1193-97$

Farnè A, Làdavas E, 2002 "Auditory peripersonal space in humans" Journal of Cognitive Neuroscience $141030-1043$

Graziano M S A, Cooke D F, 2006 "Parieto-frontal interactions, personal space, and defensive behavior" Neuropsychologia $44845-859$

Greenberg C I, Strube M J, Myers R A, 1980 "A multitrait-multimethod investigation of interpersonal distance" Journal of Nonverbal Behaviour 5 104-114

Horowitz M J, Duff D F, Stratton L O, 1964 "Body buffer zone-exploration of personal space" Archives of General Psychiatry $11651-656$

Kitagawa N, Zampini M, Spence C, 2005 "Audiotactile interactions in near and far space" Experimental Brain Research $166528-537$

Lloyd D M, 2009 "The space between us: A neurophilosophical framework for the investigation of human interpersonal space" Neuroscience \& Biobehavioral Reviews 33297 - 304

Mallenby T W, Mallenby R G, 1975 "The personal space of hard-of-hearing children with extended contact with 'normals" "British Journal of Social and Clinical Psychology 14253 - 257

Sommer R, 1959 "Studies in personal space" Sociometry $23247-260$ 


\section{PERTEPTION}

VOLUME 382009

www.perceptionweb.com

Conditions of use. This article may be downloaded from the Perception website for personal research by members of subscribing organisations. Authors are entitled to distribute their own article (in printed form or by e-mail) to up to 50 people. This PDF may not be placed on any website (or other online distribution system) without permission of the publisher. 